\title{
Effect of organic and aqueous dispersion medium on the development of polystyrene nanoparticles in nanoprecipitation method
}

\author{
Attaullah Bukhari, Ani Idris* and Madiha Atta \\ Department of Bioprocess engineering, Faculty Chemical engineering, UTM, 81310 UTM Skudai, Johor, Malaysia \\ *Corresponding Author: ani@cheme.utm.my (A. Idris)
}

\section{Article history :}

Received 28 January 2013

Revised 10 June 2013

Accepted 2 July 2013

Available online 1 August 2013

\section{GRAPHICAL ABSTRACT}

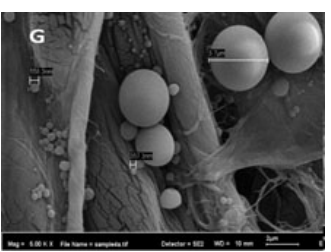

\begin{abstract}
The purpose of this study is to develop polystyrene nanoparticles using nanoprecipitation method Formation of nanoparticles from pre-polymer by nanoprecipitation is a beneficial technique involving the diffusion of polymer phase into dispersion phase. However, selection of solvent /non-solvent couple has intensive influence on nanoprecipitation of specific polymer. In this study the potential of chloroform $\left(\mathrm{CHCl}_{3}\right)$ and tetrahydrofuran (THF) is explored as the solvent for polystyrene. Effect of dispersion phase; methanol, chloroform, acetone and water have been investigated on nanoprecipitation of polystyrene. It was found that the addition of surfactants enhanced the stability of nanoparticles. Tween-40 has a more pronounced stabilizing effect than Pluronic F-68 on polystyrene nanoparticles in aqueous phase. More stable nanoparticles of polystyrene were obtained in the aqueous medium containing $2-\mathrm{g} / \mathrm{L}$ of Tween- 40 . Interface interaction of mixing phases and surface active agents affect the size and stability of nanoparticles in nanoprecipitation.
\end{abstract}

Keywords: Nanoprecipitation, Dispersion phase, Polystyrene, Nanoparticles

(C) 2014 Penerbit UTM Press. All rights reserved http://dx.doi.org/10.11113/mjfas.v10n1.66

\section{INTRODUCTION}

The word "nano" has been increasingly used in the last decade in line with its increasing applications in various research fields. Nanoparticles are defined as particulate dispersions or solid particles with a size in the range of $10-1000 \mathrm{~nm}$. It is the use and manipulation of matter at a tiny scale. At this size, atoms and molecules work differently, and provide a variety of surprising and interesting uses [1]. The expanding applications of nanoparticles integrate in fields such as: microelectronics, synthetic rubber, catalytic compounds, photographic supplies, inks and pigments, coatings and adhesives, ultrafine polishing compounds, UV absorbers for sun screens, synthetic bone, ferrofluids, optical fiber cladding, fabrics and their treatments, filtration, dental materials, surface disinfectants, diesel and fuel additives, hazardous chemical neutralizers, automotive components, electronics, scientific instruments, sports equipment, flat panel displays, drug delivery systems, and pharmaceutics [2]. Polystyrene is an extensively exploited polymer material, and the synthesis of polystyrene nanoparticles is deliberated as a model system for developing polymer nanotechnology.

A number of techniques have been reported for the development of nanomaterial depending on targeted uses
[3]. Nanoparticles have been prepared most frequently by three methods: (1) dispersion of preformed polymers; (2) polymerization of monomers; and (3) ionic gelation or coacervation of hydrophilic polymers [4]. Preparation of polymeric nanoparticles can simply be divided into: (1) polymerization of monomers (2) fabrication of nanoparticles from preformed polymer. Purification of the nanoparticles from monomers, oligomers, residual reagents like initiators, and surfactants as well as the control over the molecular properties of the polymers are challenging tasks in the polymerization technique, and sometimes require high energy as sonication and homogenization [5].

The nanoprecipitation, a promised technique (solvent displacement method) for nanoparticle manufacturing was first developed and patented by Fessi and co-workers [6,7]. Nanoprecipitation is a straightforward technique, rapid and easy to perform. The nanoparticle formation is efficient and the entire procedure is performed in only one step. In principal, it requires two solvents that are miscible. Preferably the polymer must dissolve in the first one (the solvent S), but not in the second system (the non-solvent, NS). Nanoprecipitation occurs by a rapid dissolution of the polymer when the polymer solution is added to the non-solvent. Indeed, as soon as the polymer-containing solvent has diffused into 
the dispersing medium, the polymer precipitates into nanoparticles [8].

The rapid nanoparticle formation is governed by the so-called Marangoni effect, which is due to interfacial turbulences that take place at the interface of the solvent and the non-solvent and result from complex and accumulated phenomena such as flow, diffusion and surface tension variations [9].Therefore, the nanoprecipitation technique represents a more facile, less extensive, less energy consuming as well as widely applicable technique without any additives for the manufacture of well-defined nanospheres. The formation is based on the interfacial deposition due to the displacement of a solvent against a non-solvent [7].

In 2010 Nemanja Miletic et al. prepared polystyrene nanoparticles by using non-ionic surfactants as stabilizers in nanoprecipitation technique. In order to optimize the nanoprecipitation technique, effects of various parameters such as the type and concentration of the stabilizers, the stirring speed, and the polymer concentration in the organic phase have been studied and reported by many researchers [10-12]. They observed that stirring rate and concentration of surfactant influenced the size of nanoparticles. They used the aqueous media through out as dispersion media. In this study effect of methanol, chloroform, acetone and water as dispersion media in nanoprecipiation of polystyrene is invstigated.

\section{EXPERIMENTAL}

\section{$2.1 \quad$ Materials}

Polystyreneand Tween-40 was purchased from Sigma-Aldrich. Methanol, tetrahydrofuran, chloroform, Pluronic F-68 was obtained from Merck.

\subsection{Preparation of polystyrene nanoparticles}

Solvent phases were prepared by dissolving polystyrene until concentration of 0.45 wt. \% was achieved in acetone, chloroform, tetrahydrofuran and acetonitrile discretely. Methanol, acetone, chloroform and water were used as dispersion phases. Constant concentration of surfactant (Tween-40, Pluronic F-68) $2.5 \mathrm{~g} / \mathrm{L}$ was maintained in all the dispersion phases. Each polymer phase was injected into the dispersion phase by a syringe pump at rate of $55 \mathrm{~mL} /$ hour. Continuous stirring rate was maintained at $600 \mathrm{rpm}$. Chloroform polymer phase was subjected to nanoprecipiation in water and methanol only. Immediately after the particles formation, the suspension was filtered through a metal filter of $32 \mathrm{~mm}$ mesh size and the solvent was removed from the suspension under vacuum in a rotary evaporator (IKA) at $30^{\circ} \mathrm{C}$ to a final volume of about $30 \mathrm{~mL}$. Subsequently freeze drying was performed. The morphology of polystyrene nanoparticles was studied by Field Emission Scanning electron (Supra 35 VP FESEM) micrographs.

\section{RESULTS \& DISCUSSION}

\subsection{Polymer phase}

Miscibility of the polymer in solvent is a prerequisite for nanoprecipitation. It was found that the concentration of a polymer in solvent is the limiting factor for nanoprecipitation; too high concentration of polymer is oppressive to appreciable diffusion of solvent into nonsolvent while negligible concentration owing to immiscible nature of polymer is not appreciable for nanoprecipitation. Negligible solubility attributed by acetone and acetonitrile for polystyrene is not suitable for nanoprecipitation; however polystyrene is appreciably soluble in chloroform and tetrahydrofuran. For further work tetrahydrofuran and chloroform were selected as solvent for polystyrene nanoprecipitation. The optimum concentration of polymer was found to be $0.45 \mathrm{wt}$. \% in chloroform and tetrahydrofuran.

\subsection{Effect of non-solvent (dispersion phase) nanoprecipitation}

Nanoparticles formation results of various dispersion phases are summarized in Table 1 . The results revealed that the combination of THF with acetone and water; and $\mathrm{CHCl}_{3}$ with methanol and acetone results in the formation of nanoparticles. Figure 1-4 reveals the FESEM images of the morphology of nanoparticles formed. It can be observed that the formation is remarkably dependent on the nature of dispersion phase. Development of nanoparticles was clearly observed (Fig.2, C and D) in acetone and water dispersion media in the presence of Pluronic F-68. Higher affinity and dielectric constant differences between non-solvent and solvent [13] are some of the factors that influence nanoprecipitation. Tetrahydrofuran polymer phase in the presence of non-solvent such as acetone and water significantly resulted in nanoprecipitation while polystyrene could not be precipitate in nanoparticles if the dispersion media is chloroform or methanol (Fig.1 A and Fig 2.B). This confirms that low dielectric difference between solvent and non-solvent does not favor nanoprecipitation.

However, the dielectric constant difference is not the only factor responsible for nanoprecipitation, since nanoprecipitation can also results from various phenomena that govern the diffusion of the solvent through the polymer into the non-solvent. It is therefore expected that the choice of the solvent/non-solvent coupled with the diffusion rate will affect the nanoparticles development, more than individual solvent and non-solvent characteristics such as the dielectric constant, or the Hildebrand solubility parameter, $\delta$. The affinity of the solvent for the non-solvent is an important factor. It was found the development of polystyrene nanoparticles is more pronounced if the chloroform polymer phase is precipitated in methanol (Fig. $4, \mathrm{~F})$. However, acetone and water are not useful dispersion phases for chloroform polymer phase for nanoprecipiation 
(Fig. 4, E and Fig. 6, I). Nature (dielectric constant, solubility, viscosity) of non-solvent determines nanoprecipiation of the polymer phase. Therefore, methanol led to smaller nanoparticles than water.

\subsection{Effect of surfactant}

Surfactants play a critical role in nanoprecipitation method. It was observed without surfactant the polystyrene tends to agglomerate instead of forming stable nanoparticles. In non-aqueous dispersion phase Pluronic F68 significantly stabilize nanoparticles. From Fig. 3,D it was observed that the surface of nanoparticles is not compact; prominent compressions (disc shaped) are observed on the surfaces of the polystyrene nanoparticles. The surfactant remains as part of the nanoparticles composition after their formation. Moreover Tween-40 showed remarkable influence on the morphology of the nanoparticles; the surface is more compact and uniform compared to pluronic F-68 (Fig. 5, $\mathrm{G}$ and $\mathrm{H}$ ). Nanoprecipitation of THF polymer phase was not observed in the presence of Tween-40 (Fig.6,J) and this can be attributed to dielectric constant difference between THF and water. Although the difference in the dielectric constant between the solvent/non-solvent is high, in this case the influence of surfactant tends to dominate resulting in the failure to nanoprecipitate. Surfactants play a critical role in nanoprecipitation of preformed polymer as it reduces surface tension of dispersion phase. Suitable pair of solvent/non-solvent and surfactant together affects the morphology of nanoparticles. Tween- 40 was removed successfully from nanoparticles by washing particles excessively with ethanol however Pluronic-F68 does not seem to play an important role in nanoprecipitation of the preformed polymer as it reduces surface tension of the dispersion phase.

\subsection{Effect of diffusion on size of particles}

The smaller size of PS particles in methanol is attributed to higher rate of diffusion of solvent $\left(\mathrm{CHCl}_{3}\right)$ into the non-solvent methanol. Due to the higher rate of diffusion, smaller nanoparticles are formed and the yield of transformed polymer into nanoparticles is higher. Similar observations were reported by [14]. The dielectric constant difference of methanol and chloroform is lower than that of THF and water. Increasing the flow rate of organic phase resulted in the formation of nanoparticles of larger size. This is due to the hindered interfacial turbulences at solvent and non-solvent surfaces.

Table 1.Nanoprecipitation of polystyrene at constant conc. of polymer $0.45 \mathrm{wt}$. $\%$ and $2.5 \mathrm{~g} / \mathrm{L}$ of surfactant Pluronic F-68

\begin{tabular}{|c|c|c|c|}
\hline Solvent & $\begin{array}{c}\text { Dispersion phase (Non- } \\
\text { solvent) }\end{array}$ & $\begin{array}{c}\text { Dielectric constant of non- } \\
\text { solvent }\end{array}$ & Formation of nanoparticles* \\
\hline $\mathrm{THF}^{\ddagger}$ & Methanol & 3.3 & -- \\
\hline THF & Chloroform & 4.8 & -- \\
\hline THF & Acetone & 20.7 & + \\
\hline THF & Water & 78.5 & ++ \\
\hline $\mathrm{CHCl}_{3}$ & Methanol & 3.3 & ++ \\
\hline $\mathrm{CHCl}_{3}$ & Acetone & 20.7 & + \\
\hline $\mathrm{CHCl}_{3}$ & Water & 78.5 & $\ldots$ \\
\hline
\end{tabular}

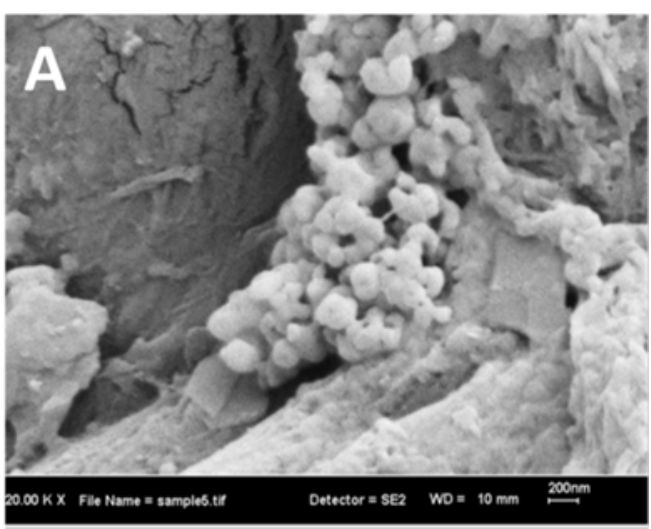

Fig. 1 FESEM micrographs of nanopolystyrene prepared by nanoprecipitation of PS in THF in non-solvent A: Chloroform

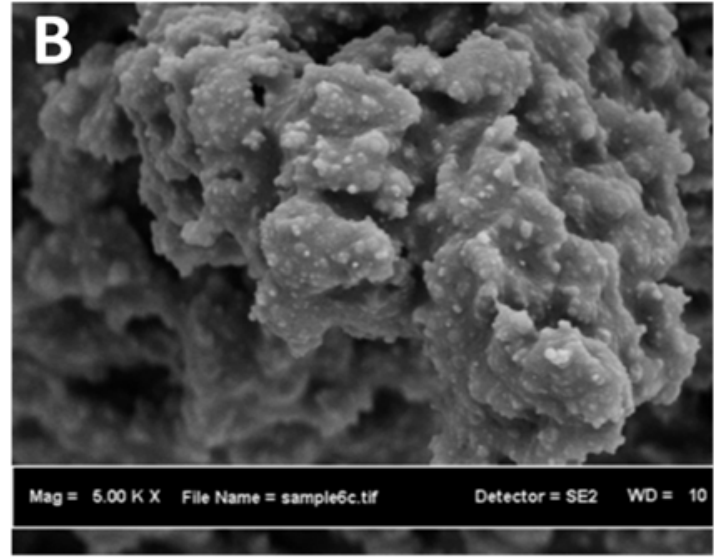

Fig. 2 FESEM micrographs of nanopolystyrene prepared by nanoprecipitation of PS in THF in non-solvent A: methanol 


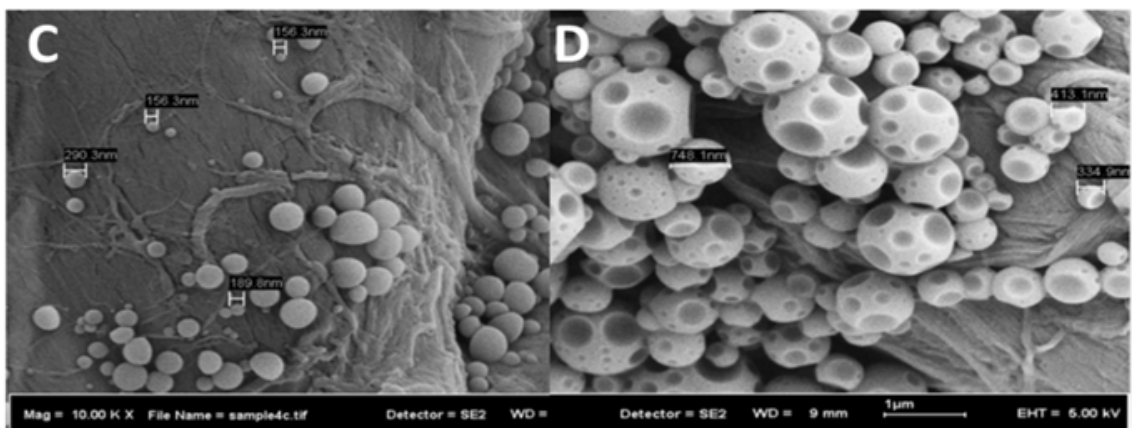

Fig. 3 FESEM micrographs of nanopolystyrene prepared by nanoprecipitation of PS in THF in non-solvent C: Acetone D: water with surfactant Pluronic-F68

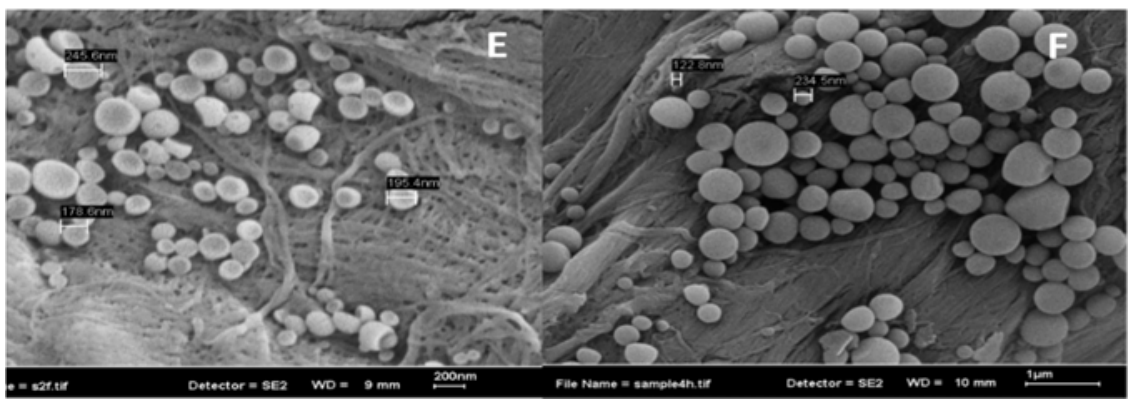

Fig. 4 FESEM micrographs of nanopolystyrene prepared by nanoprecipitation of PS in THF in non-solvent E: Water with P1 F-60, PS in Chloroform non-solvent F: Methanol

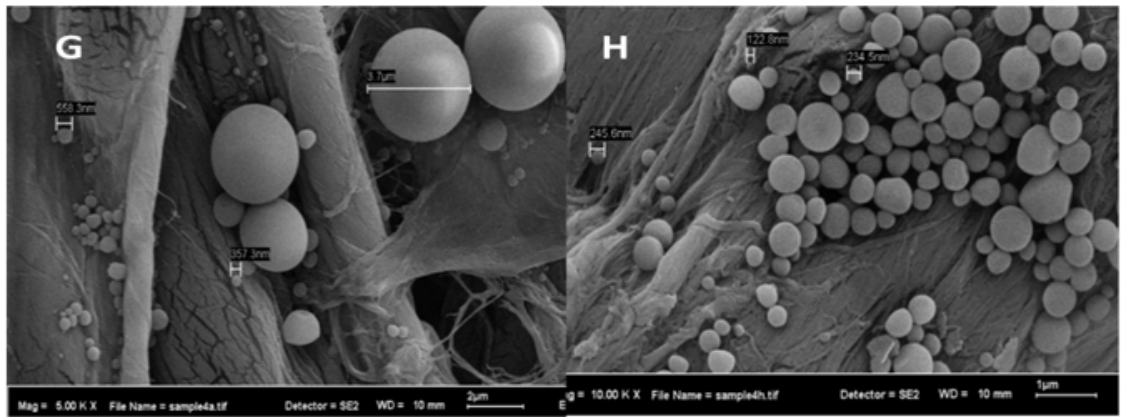

Fig. 5 FESEM micrographs of nanopolystyrene prepared by nanoprecipitation of PS in Chloroform non-solvent G: Tween-40 in water, PS in THF, H: Ploronic F-68 in water.

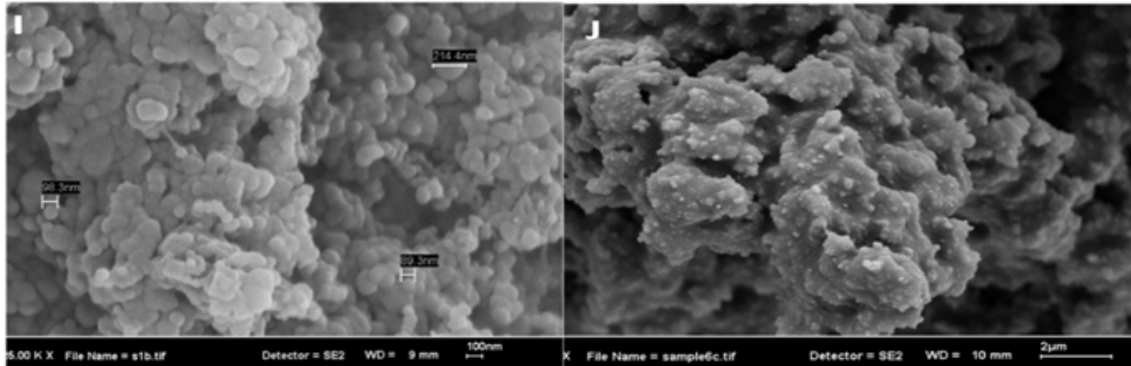

Fig. 6 FESEM micrographs of nanopolystyrene prepared by nanoprecipitation of PS in THF in non-solvent J: Tween-40 in water, PS in $\mathrm{CHCl} 3$ in I: Acetone

\section{CONCLUSION}

The choice of solvent/non-solvent and surfactants in nanoprecipitation technique has a strong influence in the size and formation of nanoparticles. The higher the dielectric difference between polymer phase and dispersion phase is helpful in nanoprecipitation depending on the type of surfactant used. Polystyrene in THF/acetone and $\mathrm{THF} /$ water in the presence of Pluronic f-68 tend to form 
small nanoparticles but when the surfactant is replaced by Tween-40, no nanoprecitation occurs. Chloroform is more potential solvent when Tween 40 in methanol or water is used. In the case of polystyrene in $\mathrm{CHCl}_{3}$ /acetone nanoparticles are not stable. I $\mathrm{n}$ addition the polymer concentration needs to be kept to 0.45 wt $\%$ or less. Flow rate of organic phase into aqueous phase and stirring rate have considerable effect on the size and stability of polystyrene nanoparticles. Polystyrene nanoparticles are more stable in aqueous phase containing Tween-40 than Pluronic f-68.

\section{ACKNOWLEDGEMENT}

This work is financially supported by Universiti Teknologi Malaysia and Ministry of Higher Education (MOHE) under vot number $02 \mathrm{H} 85$.

\section{REFERENCES}

[1] H. Xie, J. W Smith,. J. Nano 1 (2010) 8

[2] C. Buzea, I. Ivan, P. Blandino, K. Robbie. Biointer. 2 (4) (2007) 171
[3] R. G. Chaudhuri. S. Paria, Chem. Rev. 112 (2012) 2373.

[4] V. Mohanraj, Y. Chen.. Tro. J. Pharm. Res, 5 (2006) 561

[5] S. Hornig, T. Heinze, C. R. Becer, S. U. Schubertb. J. Mater. Chem. 19 (2009) 3838

[6] H. Fessi, J.P. Devissaguet, F. Puisieux, C, Thies. US. Pat. 5, 118 528, 1992.

[7] H. Fessi. F. Puisieux, J.P. Devissaguet, N.Ammoury, S. Benita. Int. J. Pharm. 55 (1989) 1.

[8] U. Bilati1, E. Alliemann, E. Doelker. Eup. J. Pharm. Sci. 24 (2005) 67.

[9] D. Q. Guerrero, E., Allemann, H, Fessi, E. Doelker. 1998. Drug Dev. Ind. Pharm. 24 (1998) 1113

[10] H.Y. Kwon, J.Y. Lee, S.W. Choi, Y. Jang, J.H. Kim, Colloids Surf A Physicochem. Eng. Aspects 182 (2001) 123.

[11] D. Q. Guerrero, H. Fessi, E. Allemann. Doelker. Int. J. Pharm.143 (1996) 133.

[12] S. W. Choi, H.Y. Kwon, W.S. Kim, J.H. Kim, ibid. 201(2002) 283.

[13] O. Thioune, H. Fessi, J.P. Devissaguet, F. Puisieux. Int. J. Pharm. 146 (1997) 233.

[14] S. Stainmesse, A.M. Orecchioni, E. Nakache. Proc. Sixth Congr. Int. Technol. Pharm. 1 (1992) 89. 